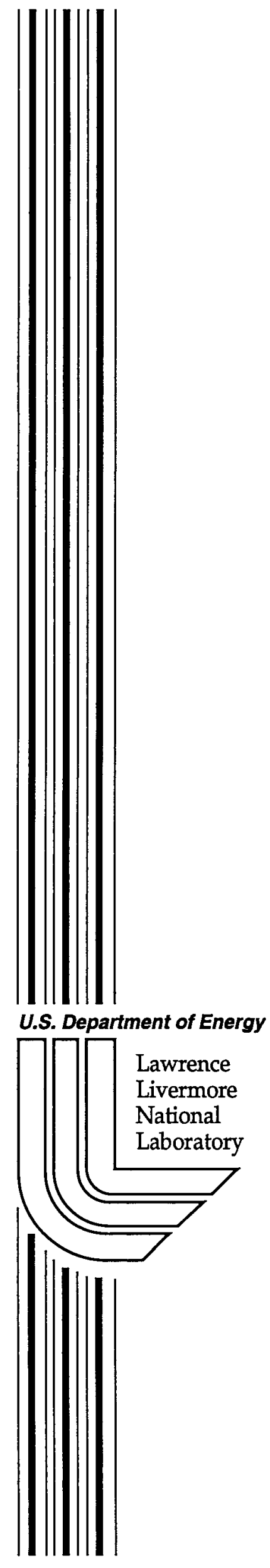

UCRL-ID-149016

\title{
Radial Reflection Diffraction Tomography Notes
}

\author{
S.K. Lehman, S.J. Norton
}

June 27, 2002 


\section{DISCLAIMER}

This document was prepared as an account of work sponsored by an agency of the United States Government. Neither the United States Government nor the University of California nor any of their employees, makes any warranty, express or implied, or assumes any legal liability or responsibility for the accuracy, completeness, or usefulness of any information, apparatus, product, or process disclosed, or represents that its use would not infringe privately owned rights. Reference herein to any specific commercial product, process, or service by trade name, trademark, manufacturer, or otherwise, does not necessarily constitute or imply its endorsement, recommendation, or favoring by the United States Government or the University of California. The views and opinions of authors expressed herein do not necessarily state or reflect those of the United States Government or the University of California, and shall not be used for advertising or product endorsement purposes.

This work was performed under the auspices of the U. S. Department of Energy by the University of California, Lawrence Livermore National Laboratory under Contract No. W-7405-Eng-48.

This report has been reproduced directly from the best available copy.

Available electronically at http://www.doc.gov/bridge

Available for a processing fee to U.S. Department of Energy

And its contractors in paper from

U.S. Department of Energy

Office of Scientific and Technical Information

P.O. Box 62

Oak Ridge, TN 37831-0062

Telephone: (865) 576-8401

Facsimile: (865) 576-5728

E-mail: reports@adonis.osti.gov

Available for the sale to the public from

U.S. Department of Commerce

National Technical Information Service

5285 Port Royal Road

Springfield, VA 22161

Telephone: (800) 553-6847

Facsimile: (703) 605-6900

E-mail: orders@ntis.fedworld.gov

Online ordering: http://www.ntis.gov/ordering.htm

OR

Lawrence Livermore National Laboratory

Technical Information Department's Digital Library

http://www.llnl.gov/tid/Library.html 


\title{
Radial Reflection Diffraction Tomography Notes
}

\author{
Sean K. Lehman \\ Lawrence Livermore National Laboratory \\ Steven J. Norton \\ Geophex
}

June 27, 2002

\begin{abstract}
We are developing the theory behind a new imaging modality which uses a single transducer rotating about its center to launch a field radially outward and collect the backscattered (reflected) field. We use diffraction tomography techniques, based upon a linearized version of the field scattering equation, to form images of the medium surrounding the transducer. As there is one transducer which both transmits the incident field and measures the backscattered field, the operation mode is multimonostatic.
\end{abstract}

\section{Introduction}

Consider a single transducer which rotates about a fixed radius, $R_{0}$, launching pulses at each angular location, $\theta$, and measuring the backscattered field. Operating in such a multimonostatic condition, we develop an imaging algorithm based upon a linearized description of the field scattering process. We summarize the operating conditions as follows:

- Transducer rotates at a fixed radius, $R_{0}$, about the origin;

- Must use frequency diversity which implies an incident pulse;

- Multimonostatic reflection operating mode in which the single transducer emits a pulse and records the backscattered field at each angular location, $\theta$;

- Geometry is that of Figure 1.

The transducer is located at $\mathrm{r}_{0}=R_{0}(\cos \theta, \sin \theta)$.

The observation point (to be reconstructed) is located at $\mathbf{r}^{\prime}=r^{\prime}\left(\cos \theta^{\prime}, \sin \theta^{\prime}\right)$ where $R_{0}<r^{\prime}$. 


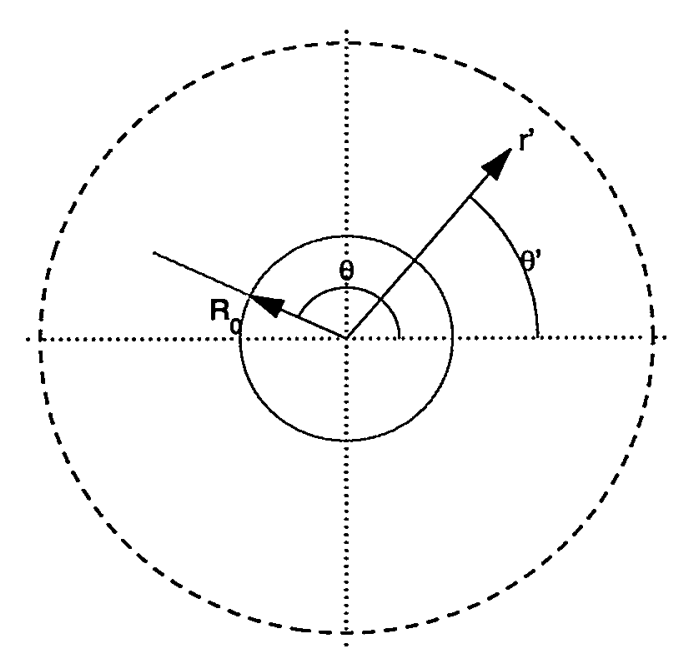

Figure 1: Radial reflection geometry

\section{Derivation of Forward Model}

Use as fundamental equation the Helmholtz equation,

$$
\left[\nabla^{2}+k^{2}(\mathbf{r})\right] u(\mathbf{r}, \omega)=-p(\mathbf{r}, \omega)
$$

where

$$
\begin{array}{ll}
\mathbf{r} \equiv(x, y)=(r, \theta) & \text { is the spatial coordinate, } \\
\omega & \text { is the temporal frequency, } \\
k(\mathbf{r}) & \text { is the wavenumber of the inhomogeneous } \\
& \text { medium surrounding the transducer, } \\
u(\mathbf{r}, \omega) & \text { is the total field, } \\
p(\mathbf{r}, \omega) & \text { is the incident pulse. }
\end{array}
$$

Add $k_{0} u(\mathbf{r}, \omega)$ to both sides of Eqn. 1 where $k_{0} \equiv \omega / v_{0}$, and move the inhomogeneous term to the right hand side:

$$
\left[\nabla^{2}+k_{0}^{2}\right] u(\mathbf{r}, \omega)=-p(\mathbf{r}, \omega)-\left[k^{2}(\mathbf{r})-k_{0}^{2}\right] u(\mathbf{r}, \omega)
$$

Define the object function as

$$
o(\mathbf{r}) \equiv \frac{k^{2}(\mathbf{r})}{k_{0}^{2}}-1
$$

and express Eqn. 2 as

$$
\left[\nabla^{2}+k_{0}^{2}\right] u(\mathbf{r}, \omega)=-p(\mathbf{r}, \omega)-k_{0}^{2} o(\mathbf{r}) u(\mathbf{r}, \omega)
$$

We may use Green's theorem to cast the differential equation of Eqn. 4 into an integral equation,

$$
u(\mathbf{r}, \omega)=\int d \mathbf{r}^{\prime} G\left(\mathbf{r}-\mathbf{r}^{\prime}, \omega\right) p\left(\mathbf{r}^{\prime}, \omega\right)+k_{0}^{2} \int d \mathbf{r}^{\prime} G\left(\mathbf{r}-\mathbf{r}^{\prime}, \omega\right) o\left(\mathbf{r}^{\prime}\right) u\left(\mathbf{r}^{\prime}\right)
$$


where we have ignored the boundary conditions and

$$
G\left(\mathbf{r}-\mathbf{r}^{\prime}, \omega\right)=\frac{e^{i k_{0}\left|\mathbf{r}-\mathbf{r}^{\prime}\right|}}{4 \pi\left|\mathbf{r}-\mathbf{r}^{\prime}\right|}
$$

The primary source is

$$
u_{i}(\mathbf{r}, \omega) \equiv \int d \mathbf{r}^{\prime} G\left(\mathbf{r}-\mathbf{r}^{\prime}, \omega\right) p\left(\mathbf{r}^{\prime}, \omega\right)
$$

so that Eqn. 5 reads

$$
u(\mathbf{r}, \omega)=u_{i}(\mathbf{r}, \omega)+k_{0}^{2} \int d \mathbf{r}^{\prime} G\left(\mathbf{r}-\mathbf{r}^{\prime}, \omega\right) o\left(\mathbf{r}^{\prime}\right) u\left(\mathbf{r}^{\prime}, \omega\right)
$$

The scattered field is defined as

$$
u_{s}(\mathbf{r}, \omega) \equiv u(\mathbf{r}, \omega)-u_{i}(\mathbf{r}, \omega)=k_{0}^{2} \int d \mathbf{r}^{\prime} G\left(\mathbf{r}-\mathbf{r}^{\prime}, \omega\right) o\left(\mathbf{r}^{\prime}\right) u\left(\mathbf{r}^{\prime}, \omega\right)
$$

Evaluate it on the measurement surface, $r_{0}=\left(R_{0}, \theta\right)$,

$$
u_{s}(\theta, \omega)=k_{0}^{2} \int d \mathbf{r}^{\prime} G\left(\mathbf{r}_{0}-\mathbf{r}^{\prime}, \omega\right) o\left(\mathbf{r}^{\prime}\right) u\left(\mathbf{r}^{\prime}, \omega\right)
$$

Let the incident field be a point source located at $\mathbf{r}_{0}$, obeying

$$
\left[\nabla^{2}+k_{0}^{2}\right] u_{i}(\mathbf{r}, \omega)=-P(\omega) \delta\left(\mathbf{r}_{0}-\mathbf{r}\right)
$$

where $P(\omega)$ is the incident pulse amplitude. NOTE: Antenna characteristics are not modeled. The incident field is then

$$
u_{i}(\mathbf{r}, \omega)=P(\omega) G\left(\mathbf{r}_{0}-\mathbf{r}, \omega\right)
$$

As is common, invoke the Born approximation,

$$
o(\mathbf{r}) \equiv \frac{k^{2}(\mathbf{r})}{k_{0}^{2}}-1 \approx 0,
$$

replace the total field by the incident field, and express Eqn. 9 as

$$
u_{s}(\theta, \omega) \approx u_{s}^{B}(\theta, \omega) \equiv P(\omega) k_{0}^{2} \int d \mathbf{r}^{\prime} G^{2}\left(\mathbf{r}_{0}-\mathbf{r}^{\prime}, \omega\right) o\left(\mathbf{r}^{\prime}\right)
$$

Eqn. 13 serves as our forward model. Express it using Eqn. 6:

$$
u_{s}^{B}(\theta, \omega)=\frac{P(\omega) k_{0}^{2}}{(4 \pi)^{2}} \int d \mathbf{r}^{\prime} \frac{e^{i 2 k_{0}\left|\mathbf{r}_{0}-\mathbf{r}^{\prime}\right|}}{\left|\mathbf{r}_{0}-\mathbf{r}^{\prime}\right|^{2}} o\left(\mathbf{r}^{\prime}\right)
$$


Define the weighted scattered field as

$$
w(\theta, 2 \omega) \equiv \frac{4 \pi}{P(\omega) k_{0}^{2}} u_{s}^{B}(\theta, \omega)=\frac{1}{4 \pi} \int d \mathbf{r}^{\prime} \frac{e^{i 2 k_{0}\left|\mathbf{r}_{0}-\mathbf{r}^{\prime}\right|}}{\left|\mathbf{r}_{0}-\mathbf{r}^{\prime}\right|^{2}} o\left(\mathbf{r}^{\prime}\right) .
$$

The division by the incident pulse spectrum is equivalent to a deconvolution in the time domain. Thus, this step performs the incident pulse deconvolution of the measured field. A more convenient expression to work with is obtained by differentiating Eqn. 15 by $k_{0}$,

$$
\begin{aligned}
\frac{d}{d k_{0}} w(\theta, 2 \omega) & =\frac{1}{4 \pi} \int d \mathbf{r}^{\prime}\left(i 2\left|\mathbf{r}_{0}-\mathbf{r}^{\prime}\right|\right) \frac{e^{i 2 k_{0}\left|\mathbf{r}_{0}-\mathbf{r}^{\prime}\right|}}{\left|\mathbf{r}_{0}-\mathbf{r}^{\prime}\right|^{2}} o\left(\mathbf{r}^{\prime}\right) \\
& =\frac{i 2}{4 \pi} \int d \mathbf{r}^{\prime} \frac{e^{i 2 k_{0}\left|\mathbf{r}_{0}-\mathbf{r}^{\prime}\right|}}{\left|\mathbf{r}_{0}-\mathbf{r}^{\prime}\right|} o\left(\mathbf{r}^{\prime}\right), \\
& =i 2 \int d \mathbf{r}^{\prime} G\left(\mathbf{r}_{0}-\mathbf{r}^{\prime}, 2 \omega\right) o\left(\mathbf{r}^{\prime}\right) .
\end{aligned}
$$

Define

$$
w^{\prime}(\theta, 2 \omega) \equiv \frac{d}{d k_{0}} w(\theta, 2 \omega)=v_{0} \frac{d}{d \omega} w(\theta, 2 \omega)
$$

We then have

$$
-\frac{i}{2} w^{\prime}(\theta, 2 \omega)=\int d \mathbf{r}^{\prime} G\left(\mathbf{r}_{0}-\mathbf{r}^{\prime}, 2 \omega\right) o\left(\mathbf{r}^{\prime}\right)
$$

\subsection{5-D Problem}

In cylindrical coordinates, let the measurement surface be at $\mathbf{r}_{0} \equiv\left(R_{0}, \theta, z_{0}\right)$ for $R_{0}$ fixed and $0 \leq \theta<2 \pi$. Thus, Eqn. 18 becomes

$$
\begin{aligned}
-\frac{i}{2} w^{\prime}(\theta, 2 \omega) & =\int_{0}^{\infty} r^{\prime} d r^{\prime} \int_{0}^{2 \pi} d \theta^{\prime} \int_{-\infty}^{\infty} d z^{\prime} G\left(\mathbf{r}_{0}-\mathbf{r}^{\prime}, 2 \omega\right) o\left(r^{\prime}, \theta^{\prime}\right), \\
& =\int_{0}^{\infty} r^{\prime} d r^{\prime} \int_{0}^{2 \pi} d \theta^{\prime} o\left(r^{\prime}, \theta^{\prime}\right) \int_{-\infty}^{\infty} d z^{\prime} G\left(\mathbf{r}_{0}-\mathbf{r}^{\prime}, 2 \omega\right) .
\end{aligned}
$$

Express $\left|\mathbf{r}_{0}-\mathbf{r}^{\prime}\right|$ in cylindrical coordinates:

$$
\begin{aligned}
\left|\mathbf{r}_{0}-\mathbf{r}^{\prime}\right|^{2} & =\left(x_{0}-x^{\prime}\right)^{2}+\left(y_{0}-y^{\prime}\right)^{2}+\left(z_{0}-z^{\prime}\right)^{2} \\
& =\left(R_{0} \cos \theta-r^{\prime} \cos \theta^{\prime}\right)^{2}+\left(R_{0} \sin \theta-r^{\prime} \sin \theta^{\prime}\right)^{2}+\left(z_{0}-z^{\prime}\right)^{2} \\
& =R_{0}^{2}+r^{2}-2 R_{0} r^{\prime} \cos \left(\theta-\theta^{\prime}\right)+\left(z_{0}-z^{\prime}\right)^{2}
\end{aligned}
$$

Defining

$$
R^{2} \equiv R_{0}^{2}+r^{2}-2 R_{0} r^{\prime} \cos \left(\theta-\theta^{\prime}\right)
$$

we note that

$$
\int_{-\infty}^{\infty} d z^{\prime} G\left(\mathbf{r}_{0}-\mathbf{r}^{\prime}, 2 \omega\right)=\frac{i}{4} H_{0}^{(1)}\left(2 k_{0} R\right) .
$$


Thus Eqn. 19 reads

$$
\begin{aligned}
-\frac{i}{2} w^{\prime}(\theta, 2 \omega) & =\frac{i}{4} \int_{0}^{\infty} r^{\prime} d r^{\prime} \int_{0}^{2 \pi} d \theta^{\prime} o\left(r^{\prime}, \theta^{\prime}\right) H_{0}^{(1)}\left(2 k_{0} R\right) \\
w^{\prime}(\theta, 2 \omega) & =-\frac{1}{2} \int_{0}^{\infty} r^{\prime} d r^{\prime} \int_{0}^{2 \pi} d \theta^{\prime} o\left(r^{\prime}, \theta^{\prime}\right) H_{0}^{(1)}\left(2 k_{0} R\right) .
\end{aligned}
$$

Hankel function expansion from G\&R (Eqn.8.530):

$$
H_{0}^{(1)}\left(k_{0} R\right)=\sum_{n=-\infty}^{\infty} J_{n}\left(k_{0} R_{0}\right) H_{n}^{(1)}\left(k_{0} r^{\prime}\right) e^{i n\left(\theta-\theta^{\prime}\right)}
$$

where $R_{0}<r^{\prime}$. Substituting this into Eqn. 22 yields

$$
w^{\prime}(\theta, 2 \omega)=-\frac{1}{2} \sum_{n=-\infty}^{\infty} \int_{0}^{\infty} r^{\prime} d r^{\prime} \int_{0}^{2 \pi} d \theta^{\prime} o\left(r^{\prime}, \theta^{\prime}\right) J_{n}\left(2 k_{0} R_{0}\right) H_{n}^{(1)}\left(2 k_{0} r^{\prime}\right) e^{i n\left(\theta-\theta^{\prime}\right)}
$$

Fourier expand the object and weighted measured field functions as follows:

$$
\begin{aligned}
o_{n}\left(r^{\prime}\right) & =\frac{1}{2 \pi} \int_{0}^{2 \pi} d \theta^{\prime} o\left(r^{\prime}, \theta^{\prime}\right) e^{-i n \theta^{\prime}} \\
o\left(r^{\prime}, \theta^{\prime}\right) & =\sum_{n=-\infty}^{\infty} o_{n}\left(r^{\prime}\right) e^{i n \theta^{\prime}}
\end{aligned}
$$

and

$$
\begin{aligned}
w_{n}^{\prime}(2 \omega) & =\frac{1}{2 \pi} \int_{0}^{2 \pi} d \theta w(\theta, 2 \omega) e^{-i n \theta} \\
w^{\prime}(\theta, 2 \omega) & =\sum_{n=-\infty}^{\infty} w_{n}^{\prime}(2 \omega) e^{i n \theta}
\end{aligned}
$$

Using Eqn. 27 to transform Eqn. 24, yields

$$
\begin{aligned}
& \frac{1}{2 \pi} \int_{0}^{2 \pi} d \theta w^{\prime}(\theta, 2 \omega) e^{-i m \theta}=-\frac{1}{4 \pi} \sum_{n=-\infty}^{\infty} J_{n}\left(2 k_{0} R_{0}\right) \int_{0}^{\infty} r^{\prime} d r^{\prime} H_{n}^{(1)}\left(2 k_{0} r^{\prime}\right) \times \\
& \underbrace{\int_{0}^{2 \pi} d \theta^{\prime} o\left(r^{\prime}, \theta^{\prime}\right) e^{-i n \theta^{\prime}}}_{\equiv 2 \pi o_{n}\left(r^{\prime}\right)} \underbrace{\int_{0}^{2 \pi} d \theta e^{i(n-m) \theta}}_{\equiv 2 \pi \delta_{m n}}
\end{aligned}
$$

Using Eqn. 25 and

$$
\frac{1}{2 \pi} \int_{0}^{2 \pi} d \theta e^{i(m-n) \theta}=\delta_{m n}
$$


reduces Eqn. 29 to

$$
w_{m}^{\prime}(2 \omega)=-\pi J_{m}\left(2 k_{0} R_{0}\right) \int_{0}^{\infty} r^{\prime} d r^{\prime} H_{m}^{(1)}\left(2 k_{0} r^{\prime}\right) o_{m}\left(r^{\prime}\right)
$$

Define the Bessel function normalized field derivative as

$$
v_{m}^{\prime}(2 \omega) \equiv-\frac{1}{\pi J_{m}\left(2 k_{0} R_{0}\right)} w_{m}^{\prime}(2 \omega)=-\frac{4 v_{0}^{3}}{J_{m}\left(2 k_{0} R_{0}\right)} \frac{d}{d \omega}\left[\frac{u_{m}(\omega)}{P(\omega) \omega^{2}}\right],
$$

where we have used Eqns. 15 and 17, and express Eqn. 30 as

$$
v_{m}^{\prime}(2 \omega)=\int_{0}^{\infty} r^{\prime} d r^{\prime} H_{m}^{(1)}\left(2 k_{0} r^{\prime}\right) o_{m}\left(r^{\prime}\right)
$$

If we assume the object function, $o(r, \theta)$, is real, we have from Eqn. 25 that

$$
o_{-m}^{*}\left(r^{\prime}\right)=o_{m}\left(r^{\prime}\right) \text {. }
$$

Using this and the property of Hankel functions,

$$
(-1)^{m} H_{-m}^{(1)}\left(2 k_{0} r\right)=H_{m}^{(1)}\left(2 k_{0} r\right),
$$

we may solve for $o_{m}(r)$. Define

$$
\begin{aligned}
P_{m}(2 \omega) & =v_{m}^{\prime}(2 \omega)+(-1)^{m} v_{-m}^{\prime *}(2 \omega), \\
& =\int_{0}^{\infty} r^{\prime} d r^{\prime} H_{m}^{(1)}\left(2 k_{0} r^{\prime}\right) o_{m}\left(r^{\prime}\right)+\int_{0}^{\infty} r^{\prime} d r^{\prime}(-1)^{m} H_{-m}^{(1) *}\left(2 k_{0} r^{\prime}\right) o_{-m}^{*}\left(r^{\prime}\right) \\
& =\int_{0}^{\infty} r^{\prime} d r^{\prime}\left[H_{m}^{(1)}\left(2 k_{0} r^{\prime}\right)+H_{m}^{(1) *}\left(2 k_{0} r^{\prime}\right)\right] o_{m}\left(r^{\prime}\right) \\
& =2 \int_{0}^{\infty} r^{\prime} d r^{\prime} J_{m}\left(2 k_{0} r^{\prime}\right) o_{m}\left(r^{\prime}\right) .
\end{aligned}
$$

Eqn. 33 is proportional to the Bessel transform of the object,

$$
o_{m}\left(2 k_{0}\right) \equiv \int_{0}^{\infty} r^{\prime} d r^{\prime} J_{m}\left(2 k_{0} r^{\prime}\right) o_{m}\left(r^{\prime}\right)
$$

so, we may express it as

$$
o_{m}\left(2 k_{0}\right)=\frac{1}{2} P_{m}(2 \omega)
$$

Explicitly expressing Eqn. 35 in terms of the measured field, we find

$$
\begin{aligned}
P_{m}(2 \omega) & =v_{m}^{\prime}(2 \omega)+(-1)^{m} v_{-m}^{*}(2 \omega), \\
& =-4 v_{0}^{3}\left[\frac{1}{J_{m}\left(2 k_{0} R_{0}\right)} \frac{d}{d \omega}\left[\frac{u_{m}(\omega)}{P(\omega) \omega^{2}}\right]+\frac{(-1)^{m}}{J_{-m}\left(2 k_{0} R_{0}\right)}\left(\frac{d}{d \omega}\left[\frac{u_{-m}(\omega)}{P(\omega) \omega^{2}}\right]\right)^{*}\right], \\
& =-\frac{4 v_{0}^{3}}{J_{m}\left(2 k_{0} R_{0}\right)} \frac{d}{d \omega}\left[\frac{1}{\omega^{2}}\left(\frac{u_{m}(\omega)}{P(\omega)}+\frac{u_{-m}^{*}(\omega)}{P^{*}(\omega)}\right)\right] .
\end{aligned}
$$


Substituting Eqn. 36 into Eqn. 35, we find the "Fourier-Bessel Diffraction Theorem" for RRDT:

$$
o_{m}\left(2 k_{0}\right)=-\frac{2 v_{0}^{3}}{J_{m}\left(2 k_{0} R_{0}\right)} \frac{d}{d \omega}\left[\frac{1}{\omega^{2}}\left(\frac{u_{m}(\omega)}{P(\omega)}+\frac{u_{-m}^{*}(\omega)}{P^{*}(\omega)}\right)\right]
$$

Using the orthogonality of Bessel functions, consider the following integral

$$
I=\int_{0}^{\infty} k d k J_{m}(k r) J_{m}\left(k r^{\prime}\right)=\frac{1}{r} \delta\left(r-r^{\prime}\right),
$$

we may invert this equation for $o_{m}(r)$ :

$$
\begin{aligned}
& 4 \int_{0}^{\infty} k_{0} d k_{0} P_{m}\left(2 k_{0} v_{0}\right) J_{m}\left(2 k_{0} r\right)=8 \int_{0}^{\infty} k_{0} d k_{0} \int_{0}^{\infty} r^{\prime} d r^{\prime} J_{m}\left(2 k_{0} r^{\prime}\right) J_{m}\left(2 k_{0} r\right) o_{m}\left(r^{\prime}\right) \\
&=8 \int_{0}^{\infty} r^{\prime} d r^{\prime} \int_{0}^{\infty} k_{0} d k_{0} J_{m}\left(2 k_{0} r^{\prime}\right) J_{m}\left(2 k_{0} r\right) o_{m}\left(r^{\prime}\right) \\
&=8 \int_{0}^{\infty} r^{\prime} d r^{\prime} \frac{1}{r^{\prime}} \delta\left(r-r^{\prime}\right) o_{m}\left(r^{\prime}\right) \\
&=8 o_{m}(r) . \\
& o_{m}(r)=2 \int_{0}^{\infty} k_{0} d k_{0} P_{m}\left(2 k_{0}\right) J_{m}\left(2 k_{0} r\right) .
\end{aligned}
$$

Substituting this into Eqn. 26 yields the reconstruction:

$$
o(r, \theta)=\sum_{m=-\infty}^{\infty} e^{i m \theta} \int_{0}^{\infty} k_{0} d k_{0} P_{m}\left(2 k_{0}\right) J_{m}\left(2 k_{0} r\right)
$$

Substitute Eqn. 36 into Eqn. 39:

$$
\begin{aligned}
o(r, \theta) & =-8 v_{0}^{3} \sum_{m=-\infty}^{\infty} e^{i m \theta} \int_{0}^{\infty} k_{0} d k_{0} \frac{d}{d \omega}\left[\frac{1}{\omega^{2}}\left(\frac{u_{m}(\omega)}{P(\omega)}+\frac{u_{-m}^{*}(\omega)}{P^{*}(\omega)}\right)\right] \frac{J_{m}\left(2 k_{0} r\right)}{J_{m}\left(2 k_{0} R_{0}\right)} \\
& =-8 v_{0} \sum_{m=-\infty}^{\infty} e^{i m \theta} \int_{0}^{\infty} \omega d \omega \frac{d}{d \omega}\left[\frac{1}{\omega^{2}}\left(\frac{u_{m}(\omega)}{P(\omega)}+\frac{u_{-m}^{*}(\omega)}{P^{*}(\omega)}\right)\right] \frac{J_{m}\left(2 k_{0} r\right)}{J_{m}\left(2 k_{0} R_{0}\right)}
\end{aligned}
$$

For later on, define the integral in Eqn. 40 as

$$
I_{m}(r) \equiv \int_{0}^{\infty} \omega d \omega \frac{d}{d \omega}\left[\frac{1}{\omega^{2}}\left(\frac{u_{m}(\omega)}{P(\omega)}+\frac{u_{-m}^{*}(\omega)}{P^{*}(\omega)}\right)\right] \frac{J_{m}\left(2 k_{0} r\right)}{J_{m}\left(2 k_{0} R_{0}\right)}
$$

and express Eqn. 40 as

$$
o(r, \theta)=-8 v_{0}^{3} \sum_{m=-\infty}^{\infty} I_{m}(r) e^{i m \theta} .
$$

We must now consider how to perform numerically the integral in Eqn. 41 . There is a potential problem with the $J_{m}^{-1}\left(2 k_{0} R_{0}\right)$ term which contains an infinite number of zeros along the real axis. 


\subsection{Extend the Integral of Eqn. 41 over the Entire Real Axis}

First some observations. The Fourier transform of the pulse is

$$
P(\omega)=\int_{0}^{\infty} d t p(t) e^{i \omega t}
$$

thus for a real pulse, we have

$$
P(-\omega)=P^{*}(\omega)
$$

The polar Fourier transform of the measured field is

$$
u_{m}(\omega)=\frac{1}{2 \pi} \int_{0}^{2 \pi} d \theta \int_{0}^{\infty} d t u(\theta, t) e^{i \omega t} e^{-i m \theta}
$$

thus we have

$$
u_{m}(-\omega)=u_{-m}^{*}(\omega) .
$$

Using these identities, we may express $I_{m}(r)$ as

$$
I_{m}(r)=\int_{-\infty}^{0} \omega d \omega \frac{d}{d \omega}\left[\frac{1}{\omega^{2}}\left(\frac{u_{m}(\omega)}{P(\omega)}+\frac{u_{-m}^{*}(\omega)}{P^{*}(\omega)}\right)\right] \frac{J_{m}\left(2 k_{0} r\right)}{J_{m}\left(2 k_{0} R_{0}\right)} .
$$

Adding Eqns 41 and 45, we have

$$
I_{m}(r)=\frac{1}{2} \int_{-\infty}^{\infty} \omega d \omega \frac{d}{d \omega}\left[\frac{1}{\omega^{2}}\left(\frac{u_{m}(\omega)}{P(\omega)}+\frac{u_{-m}^{*}(\omega)}{P^{*}(\omega)}\right)\right] \frac{J_{m}\left(2 k_{0} r\right)}{J_{m}\left(2 k_{0} R_{0}\right)}
$$

which has extended the integral over the entire real axis.

\subsection{Performing the Integral of Eqn. 46}

The difficulty in integrating Eqn. 46 is the reciprocal of the Bessel function, $J_{m}\left(2 k_{0} R_{0}\right)$, which has multiple zeros along the real axis. We circumvent this issue by continuing the integral into the complex $\omega$-plane and performing a contour integrtion. Care must be taken, however, since $J_{m}\left(2 k_{0} r\right)$ diverges in both the upper and lower half planes requiring us to express it as

$$
J_{m}\left(2 k_{0} r\right)=\frac{1}{2}\left[H_{m}^{(1)}\left(2 k_{0} r\right)+H_{m}^{(2)}\left(2 k_{0} r\right)\right] \text {. }
$$

and separating the integral into two. The one containing $H_{m}^{(1)}\left(2 k_{0} r\right)$ is closed in the upper half plane, whereas the one containing $H_{m}^{(2)}\left(2 k_{0} r\right)$ is closed in the lower half plane. We have

$$
\begin{aligned}
I_{m}(r)= & \frac{1}{4} \int_{C^{+}} \omega d \omega \frac{d}{d \omega}\left[\frac{1}{\omega^{2}}\left(\frac{u_{m}(\omega)}{P(\omega)}+\frac{u_{-m}^{*}(\omega)}{P^{*}(\omega)}\right)\right] \frac{H_{m}^{(1)}\left(2 k_{0} r\right)}{J_{m}\left(2 k_{0} R_{0}\right)}+ \\
& \frac{1}{4} \int_{C^{-}} \omega d \omega \frac{d}{d \omega}\left[\frac{1}{\omega^{2}}\left(\frac{u_{m}(\omega)}{P(\omega)}+\frac{u_{-m}^{*}(\omega)}{P^{*}(\omega)}\right)\right] \frac{H_{m}^{(2)}\left(2 k_{0} r\right)}{J_{m}\left(2 k_{0} R_{0}\right)}
\end{aligned}
$$

where the $C^{+}$contour is closed in the upper half plane, and the $C^{-}$contour is closed in the lower half plane. The $C^{+}$contour excludes the real $\omega<0$ poles but includes the real $\omega>0$ poles. The $C^{-}$contour is the opposite. These are shown in Figure 2. 

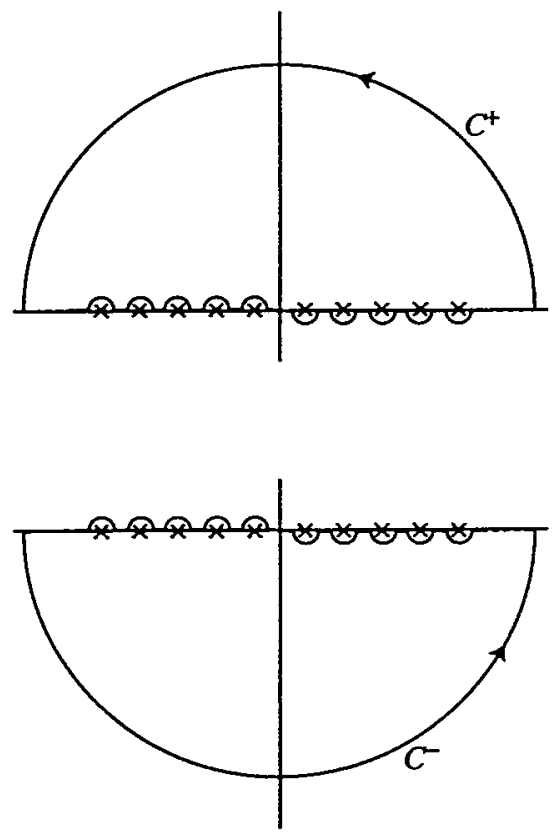

Figure 2: Integration contours.

\subsection{The Residues at the zeros of $J_{m}\left(2 k_{0} R_{0}\right)$}

In anticipation of requiring the residues of $1 / J_{m}\left(2 k_{0} R_{0}\right)$ at the zeros of the Bessel function, we compute them as follows,

$$
\begin{aligned}
j_{m n}=\operatorname{Res}_{z \rightarrow \alpha_{m n}}\left\{\frac{1}{J_{m}(z)}\right\} & =\lim _{z \rightarrow \alpha_{m n}} \frac{z-\alpha_{m n}}{J_{m}(z)} \\
& =\lim _{z \rightarrow \alpha_{m n}} \frac{2}{J_{m-1}(z)-J_{m+1}(z)} \\
& =\frac{2}{J_{m-1}\left(\alpha_{m n}\right)-J_{m+1}\left(\alpha_{m n}\right)}
\end{aligned}
$$

where $\alpha_{m n}$ is the $n^{\text {th }}$ zero of the $m^{\text {th }}$ Bessel function, and $j_{m n}$ is the residue at the positive pole. For negative poles, we have

$$
\begin{aligned}
k_{m n}=\operatorname{Res}_{z \rightarrow-\alpha_{m n}}\left\{\frac{1}{J_{m}(z)}\right\} & =\lim _{z \rightarrow-\alpha_{m n}} \frac{z+\alpha_{m n}}{J_{m}(z)} \\
& =\lim _{z \rightarrow-\alpha_{m n}} \frac{2}{J_{m-1}(z)-J_{m+1}(z)} \\
& =\frac{2}{J_{m-1}\left(-\alpha_{m n}\right)-J_{m+1}\left(-\alpha_{m n}\right)}
\end{aligned}
$$


Use $J_{m}(-z)=(-1)^{m} J_{m}(z)$ to find the $k_{m n}$ and $j_{m n}$ are related via

$$
\begin{aligned}
k_{m n} & =\frac{2}{(-1)^{m-1} J_{m-1}\left(\alpha_{m n}\right)-(-1)^{m+1} J_{m+1}\left(\alpha_{m n}\right)} \\
& =\frac{(-1)^{m+1} 2}{J_{m-1}\left(\alpha_{m n}\right)-J_{m+1}\left(\alpha_{m n}\right)} \\
& =(-1)^{m+1} j_{m n} .
\end{aligned}
$$

\subsection{Perform Complex Integration}

Replace the contour integrals of Eqn. 48 by the sum of the residues,

$$
\begin{aligned}
I_{m}(r)= & i \pi \sum_{n=1}^{\infty} \underset{2 k_{0} R_{0} \rightarrow \alpha_{m n}}{\operatorname{Res}}\left\{\omega \frac{d}{d \omega}\left[\frac{1}{\omega^{2}}\left(\frac{u_{m}(\omega)}{P(\omega)}+\frac{u_{-m}^{*}(\omega)}{P^{*}(\omega)}\right)\right] \frac{H_{m}^{(1)}\left(2 k_{0} r\right)}{J_{m}\left(2 k_{0} R_{0}\right)}\right\} \\
& -\frac{i \pi}{2} \sum_{n=1}^{\infty} \underset{2 k_{0} R_{0} \rightarrow-\alpha_{m n}}{\operatorname{Res}}\left\{\omega \frac{d}{d \omega}\left[\frac{1}{\omega^{2}}\left(\frac{u_{m}(\omega)}{P(\omega)}+\frac{u_{-m}^{*}(\omega)}{P^{*}(\omega)}\right)\right] \frac{H_{m}^{(1)}\left(2 k_{0} r\right)}{J_{m}\left(2 k_{0} R_{0}\right)}\right\} \\
& -\frac{i \pi}{2} \sum_{n=1}^{\infty} \underset{2 k_{0} R_{0} \rightarrow \alpha_{m n}}{\operatorname{Res}}\left\{\omega \frac{d}{d \omega}\left[\frac{1}{\omega^{2}}\left(\frac{u_{m}(\omega)}{P(\omega)}+\frac{u_{-m}^{*}(\omega)}{P^{*}(\omega)}\right)\right] \frac{H_{m}^{(2)}\left(2 k_{0} r\right)}{J_{m}\left(2 k_{0} R_{0}\right)}\right\} \\
& +i \pi \sum_{n=1}^{\infty} \underset{2 k_{0} R_{0} \rightarrow-\alpha_{m n}}{\operatorname{Res}}\left\{\omega \frac{d}{d \omega}\left[\frac{1}{\omega^{2}}\left(\frac{u_{m}(\omega)}{P(\omega)}+\frac{u_{-m}^{*}(\omega)}{P^{*}(\omega)}\right)\right] \frac{H_{m}^{(2)}\left(2 k_{0} r\right)}{J_{m}\left(2 k_{0} R_{0}\right)}\right\}
\end{aligned}
$$

Note

$$
\begin{aligned}
& 2 k_{0} R_{0} \rightarrow \alpha_{m n} \Rightarrow k_{0}=\frac{\alpha_{m n}}{2 R_{0}} \quad \text { and } \quad \omega=\frac{\alpha_{m n} v_{0}}{2 R_{0}} \\
& 2 k_{0} R_{0} \rightarrow-\alpha_{m n} \Rightarrow k_{0}=-\frac{\alpha_{m n}}{2 R_{0}} \quad \text { and } \quad \omega=-\frac{\alpha_{m n} v_{0}}{2 R_{0}}
\end{aligned}
$$

Define

$$
\begin{aligned}
\omega_{m n} & \equiv \frac{\alpha_{m n} v_{0}}{2 R_{0}} \\
\hat{r} & \equiv \frac{r}{R_{0}} \\
A_{m}(\omega) & \equiv \frac{d}{d \omega}\left[\frac{1}{\omega^{2}}\left(\frac{u_{m}(\omega)}{P(\omega)}+\frac{u_{-m}^{*}(\omega)}{P^{*}(\omega)}\right)\right] .
\end{aligned}
$$


Substitute Eqns. 49 and 51 into Eqn. 52 and use the above definitions,

$$
\begin{aligned}
I_{m}(r)= & i \pi \sum_{n=1}^{\infty} j_{m n} \omega_{m n} A_{m}\left(\omega_{m n}\right) H_{m}^{(1)}\left(\alpha_{m n} \hat{r}\right) \\
& -\frac{i \pi}{2} \sum_{n=1}^{\infty}(-1)^{m+1} j_{m n} \omega_{m n} A_{m}\left(-\omega_{m n}\right) H_{m}^{(1)}\left(-\alpha_{m n} \hat{r}\right) \\
& -\frac{i \pi}{2} \sum_{n=1}^{\infty} j_{m n} \omega_{m n} A_{m}\left(\omega_{m n}\right) H_{m}^{(2)}\left(\alpha_{m n} \hat{r}\right) \\
& +i \pi \sum_{n=1}^{\infty}(-1)^{m+1} j_{m n} \omega_{m n} A_{m}\left(-\omega_{m n}\right) H_{m}^{(2)}\left(-\alpha_{m n} \hat{r}\right)
\end{aligned}
$$

Note, using $H_{m}^{(1,2)}(-z)=(-1)^{m} H_{m}^{(1,2)}(z)$, we have

$$
(-1)^{m+1} H_{m}^{(1,2)}(-z)=(-1)^{2 m+1} H_{m}^{(1,2)}(z)=-H_{m}^{(1,2)}(z) .
$$

So we have

$$
\begin{aligned}
I_{m}(r)= & i \pi \sum_{n=1}^{\infty} j_{m n} \omega_{m n} A_{m}\left(\omega_{m n}\right) H_{m}^{(1)}\left(\alpha_{m n} \hat{r}\right) \\
& +\frac{i \pi}{2} \sum_{n=1}^{\infty} j_{m n} \omega_{m n} A_{m}\left(-\omega_{m n}\right) H_{m}^{(1)}\left(\alpha_{m n} \hat{r}\right) \\
& -\frac{i \pi}{2} \sum_{n=1}^{\infty} j_{m n} \omega_{m n} A_{m}\left(\omega_{m n}\right) H_{m}^{(2)}\left(\alpha_{m n} \hat{r}\right) \\
& -i \pi \sum_{n=1}^{\infty} j_{m n} \omega_{m n} A_{m}\left(-\omega_{m n}\right) H_{m}^{(2)}\left(\alpha_{m n} \hat{r}\right) \\
= & i \pi \sum_{n=1}^{\infty} j_{m n} \omega_{m n}\left(A_{m}\left(\omega_{m n}\right)+\frac{1}{2} A_{m}\left(-\omega_{m n}\right)\right) H_{m}^{(1)}\left(\alpha_{m n} \hat{r}\right) \\
& -\left(\frac{1}{2} A_{m}\left(\omega_{m n}\right)+A_{m}\left(-\omega_{m n}\right)\right) H_{m}^{(2)}\left(\alpha_{m n} \hat{r}\right) \\
= & \frac{i \pi}{2} \sum_{n=1}^{\infty} j_{m n} \omega_{m n}\left[\left(A_{m}\left(\omega_{m n}\right)-A_{m}\left(-\omega_{m n}\right)\right) J_{m}\left(\alpha_{m n} \hat{r}\right)\right. \\
& \left.+3 i\left(A_{m}\left(\omega_{m n}\right)+A_{m}\left(-\omega_{m n}\right)\right) N_{m}\left(\alpha_{m n} \hat{r}\right)\right] .
\end{aligned}
$$

Finally, we note that $P(\omega)$ and $u_{m}(\omega)$ are band limited to $\omega \in\left[\omega_{\min }, \omega_{\max }\right] \equiv \Omega$.

$$
\begin{aligned}
I_{m}(r)= & \frac{i \pi}{2} \sum_{\alpha_{m n} \in \Omega} j_{m n} \omega_{m n}\left[\left(A_{m}\left(\omega_{m n}\right)-A_{m}\left(-\omega_{m n}\right)\right) J_{m}\left(\alpha_{m n} \hat{r}\right)\right. \\
& \left.+3 i\left(A_{m}\left(\omega_{m n}\right)+A_{m}\left(-\omega_{m n}\right)\right) N_{m}\left(\alpha_{m n} \hat{r}\right)\right]
\end{aligned}
$$


Substituting Eqn. 57 back into Eqn. 40, we have the final form for the reconstruction

$$
\begin{aligned}
o(r, \theta)= & -4 i \pi v_{0} \sum_{m=-\infty}^{\infty} e^{i m \theta} \sum_{\alpha_{m n} \in \Omega} j_{m n} \omega_{m n}\left[\left(A_{m}\left(\omega_{m n}\right)-A_{m}\left(-\omega_{m n}\right)\right) J_{m}\left(\alpha_{m n} \hat{r}\right)\right. \\
& \left.+3 i\left(A_{m}\left(\omega_{m n}\right)+A_{m}\left(-\omega_{m n}\right)\right) N_{m}\left(\alpha_{m n} \hat{r}\right)\right] .
\end{aligned}
$$

\section{Sanity Check: Test Reconstruction with a Symmetric Object}

The object is independent of angle,

$$
o(r, \theta) \equiv o(r)
$$

Then the polar transform of the field is

$$
u_{m}(\omega)=\frac{1}{2 \pi} \int_{0}^{2 \pi} d \theta u(\omega) e^{-i m \theta}=u(\omega) \delta_{m 0}
$$

Then Eqn. 60 reduces to

$$
\begin{aligned}
o(r)= & -4 i \pi v_{0} \sum_{\alpha_{0 n} \in \Omega} j_{0 n} \omega_{0 n}\left[\left(A_{0}\left(\omega_{0 n}\right)-A_{0}\left(-\omega_{0 n}\right)\right) J_{0}\left(\alpha_{0 n} \hat{r}\right)\right. \\
& \left.+3 i\left(A_{0}\left(\omega_{0 n}\right)+A_{0}\left(-\omega_{0 n}\right)\right) N_{0}\left(\alpha_{0 n} \hat{r}\right)\right]
\end{aligned}
$$

Note,

$$
A_{0}\left( \pm \omega_{0 n}\right) \equiv \frac{d}{d \omega}\left[\frac{1}{\omega^{2}}\left(\frac{u(\omega)}{P(\omega)}+\frac{u^{*}(\omega)}{P^{*}(\omega)}\right)\right]_{\omega= \pm \omega_{0 n}}=2 \frac{d}{d \omega}\left[\frac{1}{\omega^{2}} \operatorname{Re}\left\{\frac{u(\omega)}{P(\omega)}\right\}\right]_{\omega= \pm \omega_{0 n}}
$$

Using the symmetry properties of Eqns. 43 and 44, we find

$$
A_{0}\left(\omega_{0 n}\right)-A_{0}\left(-\omega_{0 n}\right)=0
$$

which reduces the reconstruction to

$$
o(r)=\frac{48 \pi v_{0}^{2}}{R_{0}} \sum_{\alpha_{0 n} \in \Omega} \frac{\alpha_{0 n} N_{0}\left(\alpha_{0 n} \frac{r}{R_{0}}\right)}{J_{-1}\left(\alpha_{0 n}\right)-J_{1}\left(\alpha_{0 n}\right)} \frac{d}{d \omega}\left[\frac{1}{\omega^{2}} \operatorname{Re}\left\{\frac{u(\omega)}{P(\omega)}\right\}\right]_{\omega=\omega_{0 n}},
$$

where we have substituted,

$$
\begin{aligned}
\omega_{0 n} & =\frac{\alpha_{0 n} v_{0}}{2 R_{0}} \\
\hat{r} & =\frac{r}{R_{0}} \\
j_{0 n} & =\frac{2}{J_{-1}\left(\alpha_{0 n}\right)-J_{1}\left(\alpha_{0 n}\right)} .
\end{aligned}
$$

The reconstruction is pure real. 


\section{Fourier \& Fourier-Bessel Transforms, \& Fourier Diffraction Theorem}

The derivations used herein are based upon the Fourier-Bessel transform pair:

$$
\begin{aligned}
& F_{m}(k)=\frac{1}{2 \pi} \int_{0}^{2 \pi} d \theta \int_{0}^{\infty} r d r f(r, \theta) e^{-i m \theta} J_{m}(k r), \\
& f(r, \theta)=\sum_{m=-\infty}^{\infty} \int_{0}^{\infty} k d k F_{m}(k) e^{i m \theta} J_{m}(k r)
\end{aligned}
$$

The Cartesian Fourier transform pair,

$$
\begin{aligned}
F\left(k_{x}, k_{y}\right) & =\int_{-\infty}^{\infty} d x \int_{-\infty}^{\infty} d y F(x, y) e^{-i\left(k_{x} x+k_{y} y\right)} \\
f(x, y) & =\frac{1}{(2 \pi)^{2}} \int_{-\infty}^{\infty} d k_{x} \int_{-\infty}^{\infty} d k_{y} F\left(k_{x}, k_{y}\right) e^{i\left(k_{x} x+k_{y} y\right)}
\end{aligned}
$$

can be cast into polar coordinates using the following change of variables,

\begin{tabular}{|l|l|}
\hline$x=r \cos \theta$, & $k_{x}=k \cos \phi$, \\
$y=r \sin \theta$, & $k_{y}=k \sin \phi$, \\
\hline
\end{tabular}

to yield

$$
\begin{aligned}
F(k, \phi) & =\int_{0}^{2 \pi} d \theta \int_{0}^{\infty} r d r f(r, \theta) e^{-i k r \cos (\theta-\phi)} \\
f(r, \theta) & =\frac{1}{(2 \pi)^{2}} \int_{0}^{2 \pi} d \phi \int_{0}^{\infty} k d k F(k, \phi) e^{i k r \cos (\theta-\phi)}
\end{aligned}
$$

To determine the relationship between $F(k, \phi)$ and $F_{m}(k)$, substitute the expansion

$$
e^{-i k r \cos \theta}=\sum_{m=-\infty}^{\infty}(-i)^{m} J_{m}(k r) e^{i m \theta}
$$

into Eqn. 69

$$
\begin{aligned}
F(k, \phi) & =\int_{0}^{2 \pi} d \theta \int_{0}^{\infty} r d r f(r, \theta)\left[\sum_{m=-\infty}^{\infty}(-i)^{m} J_{m}(k r) e^{i m(\phi-\theta)}\right] \\
& =\sum_{m=-\infty}^{\infty}(-i)^{m} e^{i m \phi} \int_{0}^{2 \pi} d \theta \int_{0}^{\infty} r d r f(r, \theta) J_{m}(k r) e^{-i m \theta} \\
& =\sum_{m=-\infty}^{\infty}(-i)^{m} e^{i m \phi} F_{m}(k) .
\end{aligned}
$$


Using $\frac{1}{2 \pi} \int_{0}^{2 \pi} d \theta e^{i(m-n) \theta}=\delta_{m n}$, we may invert Eqn. 71 to find

$$
F_{m}(k)=\frac{(-i)^{-m}}{2 \pi} \int_{0}^{2 \pi} d \phi F(k, \phi) e^{-i m \phi}
$$

\subsection{The RRDT Fourier Diffraction Theorem}

Substitute Eqn. 37 into Eqn. 71 to obtain the Fourier Diffraction Theorem for RRDT,

$$
\begin{aligned}
O\left(2 k_{0}, \phi\right) & =\sum_{m=-\infty}^{\infty}(-i)^{m} e^{i m \phi} o_{m}\left(2 k_{0}\right), \\
& =-2 v_{0}^{3} \sum_{m=-\infty}^{\infty} \frac{(-i)^{m} e^{i m \phi}}{J_{m}\left(2 k_{0} R_{0}\right)} \frac{d}{d \omega}\left[\frac{1}{\omega^{2}}\left(\frac{u_{m}(\omega)}{P(\omega)}+\frac{u_{-m}^{*}(\omega)}{P^{*}(\omega)}\right)\right] .
\end{aligned}
$$

This is shown graphically in Figure 3. 


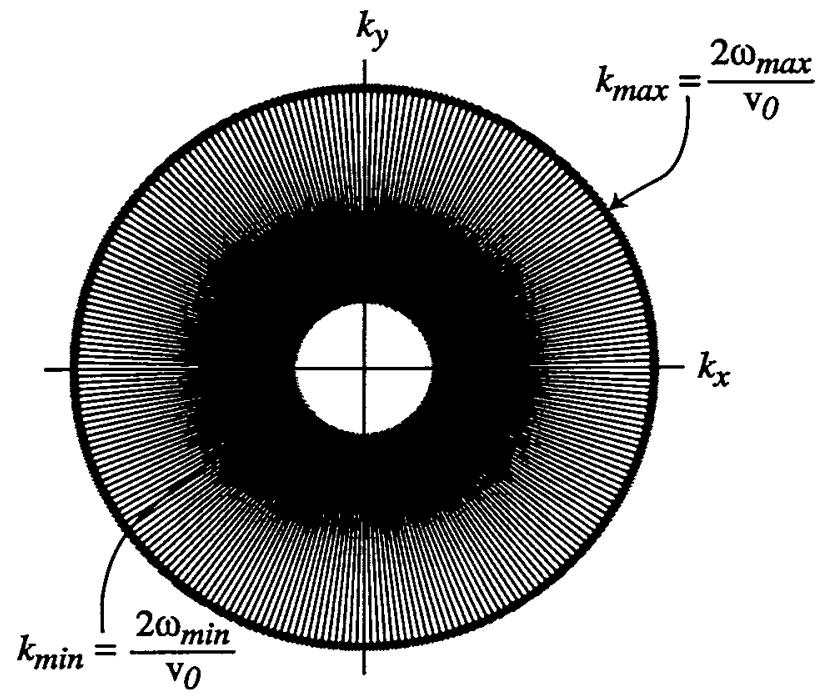

(a)

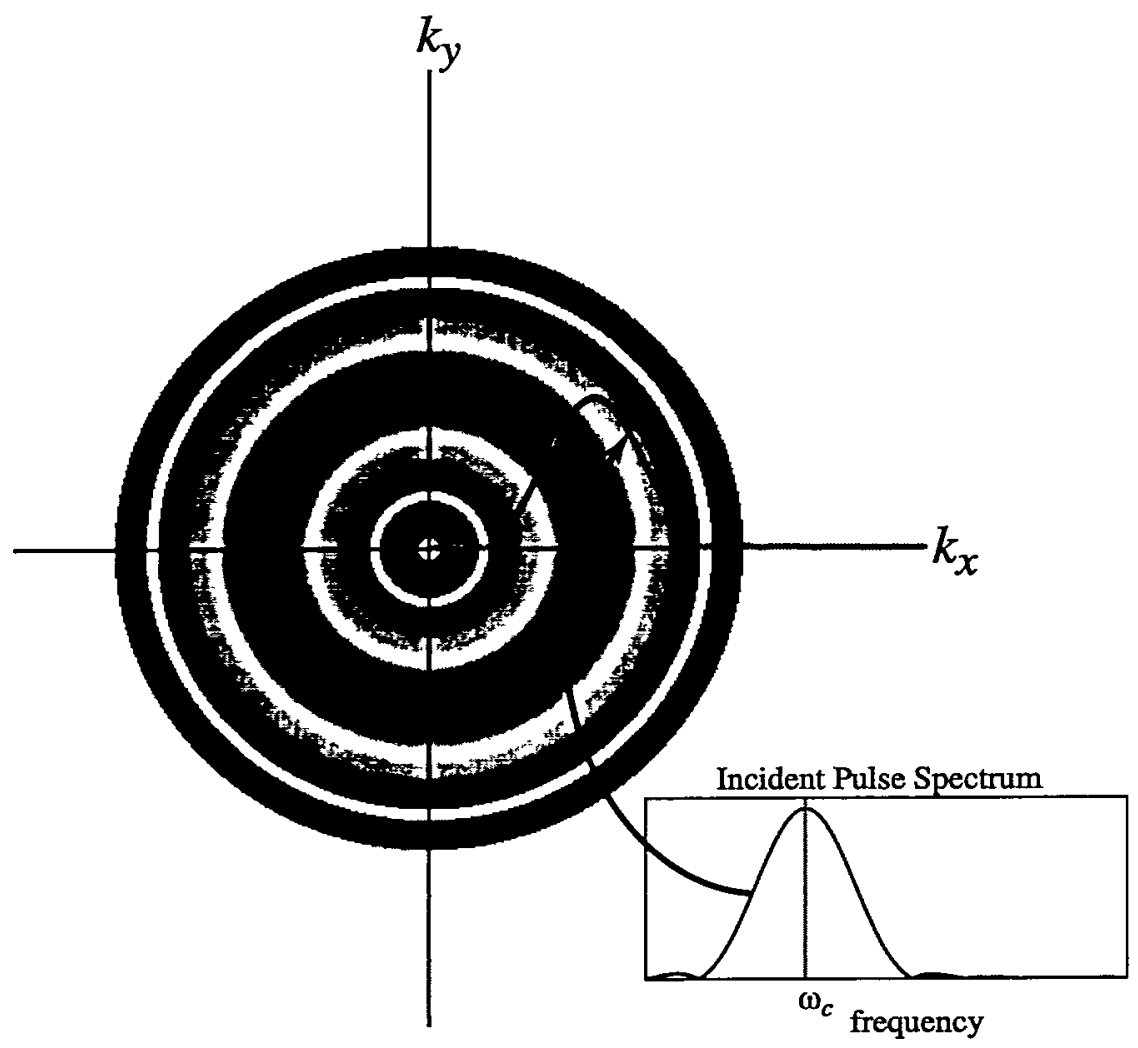

(b)

Figure 3: Radial reflection Fourier diffraction theorem. 